Published in "Cognitive Psychology 77: 1-19, 2015"

which should be cited to refer to this work.

\title{
Human short-term spatial memory: Precision predicts capacity
}

\author{
Pamela Banta Lavenex ${ }^{\mathrm{a}, *}$, Valérie Boujon ${ }^{\mathrm{b}}$, Angélique Ndarugendamwo ${ }^{\mathrm{b}}$, \\ Pierre Lavenex ${ }^{\mathrm{a}, \mathrm{b}}$ \\ ${ }^{a}$ Laboratory for Experimental Research on Behavior, Institute of Psychology, University of Lausanne, 1015 Lausanne, Switzerland \\ ${ }^{\mathrm{b}}$ Laboratory of Brain and Cognitive Development, Department of Medicine E' Fribourg Center for Cognition, University of \\ Fribourg, 1700 Fribourg, Switzerland
}

\begin{abstract}
Here, we aimed to determine the capacity of human short-term memory for allocentric spatial information in a real-world setting. Young adults were tested on their ability to learn, on a trial-unique basis, and remember over a 1 -min interval the location(s) of $1,3,5$, or 7 illuminating pads, among 23 pads distributed in a $4 \mathrm{~m} \times 4 \mathrm{~m}$ arena surrounded by curtains on three sides. Participants had to walk to and touch the pads with their foot to illuminate the goal locations. In contrast to the predictions from classical slot models of working memory capacity limited to a fixed number of items, i.e., Miller's magical number 7 or Cowan's magical number 4 , we found that the number of visited locations to find the goals was consistently about 1.6 times the number of goals, whereas the number of correct choices before erring and the number of errorless trials varied with memory load even when memory load was below the hypothetical memory capacity. In contrast to resource models of visual working memory, we found no evidence that memory resources were evenly distributed among unlimited numbers of items to be remembered. Instead, we found that memory for even one individual location was imprecise, and that memory performance for one location could be used to predict memory performance for multiple locations. Our findings are consistent with a theoretical model suggesting that the precision of the memory for individual locations might determine the capacity of human shortterm memory for spatial information.
\end{abstract}

* Corresponding author at: Laboratory for Experimental Research on Behavior, Institute of Psychology, University of Lausanne, Géopolis, 1015 Lausanne, Switzerland. Fax: +41 216923260 .

E-mail address: pamela.bantalavenex@unil.ch (P. Banta Lavenex). 


\section{Introduction}

Historically, two constructs have been used to describe the processing of memories over short periods: short-term memory and working memory (Baddeley, 2003; Becker \& Morris, 1999; Cowan, 2008; Unsworth \& Engle, 2007). Following Baddeley and Hitch's model of working memory (Baddeley \& Hitch, 1974), numerous studies investigated the capacity of the so-called "visuo-spatial sketchpad". Paradigms used to study visuo-spatial working memory processes typically assess participants' abilities to remember "what" they saw (visual memory), "where" they saw it (spatial memory), or "what" they saw "where" (visuo-spatial memory). Participants are usually shown one to several stimuli on a screen or a piece of paper placed in front of them, and after a short delay (one to several seconds) are asked to recall what the specific stimulus was, or whether there was a change in the location of one of the stimuli. Estimates of visuo-spatial short-term memory capacity have typically revealed an upper limit of $4 \pm 1$ items (see (Cowan, 2001) for a comprehensive review and discussion of human mental storage capacity).

Other paradigms designed to evaluate visuo-spatial memory capacity assess participants' serial recall of spatial locations. Participants must repeat a previously-viewed, serially-demonstrated target sequence. Investigations assessing serial spatial memory span have implemented the Corsi block-tapping task with nine blocks (CBT; (Corsi, 1972)), or target locations presented on a screen (Avons, 2007; Fagot \& De Lillo, 2011; Parmentier, Elford, \& Mayberry, 2005). The length of the sequence repeated correctly represents the spatial span; immediate span for block-tapping was originally reported to be 4.9 for normal young adults (average age: 28.1 years; (Corsi, 1972)). Replications of the original task revealed an average spatial memory span around $6 \pm 1$ for normal controls (Berch, Krikorian, \& Huha, 1998; Farrell Pagulayan, Busch, Medina, Bartok, \& Krikorian, 2006). Recently, Piccardi and colleagues developed the Walking Corsi Test (WalCT), a large-scale version of the CBT (scale 1:10), in which participants have to walk and reach different locations in a real environment (Piccardi et al., 2008). Normative data in healthy young adults revealed a serial spatial memory span ranging from 5.96 to 7.37 with the CBT, and from 5.61 to 7.93 in the WalCT, depending on the delay between presentation and recall (Piccardi et al., 2013). Estimates of the serial spatial memory span are thus more in agreement with Miller's magical number $7 \pm 2$, as the limit of our immediate memory capacity for individual items or chunks of information (Miller, 1956).

It is important to note that, although those studies assessed visuo-spatial memory, they did not assess allocentric spatial memory. Indeed, the brain can represent object locations, whether they are real objects in the real world, or a visual array presented on a screen or a piece of paper, via distinct spatial representation systems (Banta Lavenex \& Lavenex, 2009; Burgess, 2006; O'Keefe \& Nadel, 1978). Over the short term, and when the observer's position is fixed in relation to a real or graphically-presented object array, egocentric (body-centered or viewpoint-dependent) coding is the most reliable, and allocentric (world-centered or viewpoint-independent) encoding unnecessary (Banta Lavenex et al., 2011). However, once the observer begins to move in the world, allocentric spatial coding becomes critical to spatial memory processing (Banta Lavenex, Colombo, Ribordy Lambert, \& Lavenex, 2014; Banta Lavenex \& Lavenex, 2009; Burgess, 2006; O'Keefe \& Nadel, 1978). Over the years, a number of researchers have investigated human short-term visuo-spatial memory using tasks distinguishing different spatial frames of reference (Abrahams, Pickering, Polkey, \& Morris, 1997; Burgess, Spiers, \& Paleologou, 
2004; Holdstock, Mayes, Cezayirli, Aggleton, \& Roberts, 1999; Holdstock et al., 2000; Morris, Pickering, Abrahams, \& Feigenbaum, 1996; Mou, McNamara, Valiquette, \& Rump, 2004; Simons \& Wang, 1998; Wang \& Simons, 1999). Whereas some of these studies aspired to determine whether participants could detect a change in the position of one object, in an array of several objects, and whether that objectlocation memory was influenced by the availability of egocentric and/or allocentric frames of reference (Burgess et al., 2004; Mou et al., 2004; Simons \& Wang, 1998; Wang \& Simons, 1999), others reported the number of errors in order to compare the performance of brain-lesioned patients with that of control subjects (Abrahams et al., 1997; Holdstock et al., 1999, 2000; Morris et al., 1996). However, none of these studies provided estimates of human allocentric spatial memory capacity in a real-world setting.

In contrast, we previously investigated whether humans' access to egocentric or allocentric spatial frames of reference affects their memory capacity in a short-term spatial memory task performed in the real world (Banta Lavenex et al., 2011). Subjects were asked to play the Memory game (a.k.a. the Concentration game) without an opponent: subjects were instructed to turn two cards sequentially (i.e., one card and then a second card), and remove these cards from the game if they matched. If the images did not match, subjects had to return the cards to their face down position in the exact location where they found them. The test was complete when all pairs were found. Subjects performed the task in four different conditions that varied subjects' ability to rely on egocentric and/or allocentric frames of reference for the elaboration of a spatial representation of the image locations: A. Stationary: both the game tray and the subject remained in the same position throughout the game. B. Subject and tray rotate: both the subject and the game tray rotated $90^{\circ}$ in the same direction between pair of choices. C. Subject rotates: the game tray remained in the same position, but the subject rotated $90^{\circ}$ around the apparatus between every pair of choices. D. Tray rotates: the subject remained in the same position, but rotated the game tray $90^{\circ}$ between every pair of choices. We found that subjects' short-term spatial memory capacity was greater when egocentric information could be used to remember the location of identified images (A and B), than when only allocentric information could be used ( $C$ and $D$ ). With 8 pairs of images (16 locations), individuals retained on average 0.58 more items in memory in egocentric conditions (3.33) than in allocentric conditions (2.75) (Effect size: $\left.\left(\mu_{2}-\mu_{1}\right) / \sigma=1.52 ; t_{(19)}=8.434, p<0.0001\right)$. Similarly, with 12 pairs of images (24 locations), individuals retained on average 0.92 more items in memory in egocentric conditions (3.91) than in allocentric conditions (2.99) (Effect size: $2.07 ; t_{(19)}=10.109, p<0.0001$ ). Surprisingly, in egocentric conditions, individuals retained on average 0.58 more items in memory with 12 pairs of images (3.91) than with 8 pairs of images (3.33) (Effect size: $1.42 ; t_{(19)}=2.118, p=0.048$ ). In allocentric conditions, there was no significant difference between 8 pairs (2.75) and 12 pairs of images (2.99) (Effect size: $\left.0.59 ; t_{(19)}=0.867, p=0.397\right)$. Although these findings seem consistent with Cowan's magical number 4 as the upper limit for human short-term memory capacity (Cowan, 2001), Cowan's conception of memory capacity does not enable us to explain why we found different short-term spatial memory capacities for the same image sets depending on whether the information was represented in egocentric and/or allocentric frames of reference, or why there was an apparent increase in memory capacity when there was a larger number of items to remember.

It is thus interesting to consider the entirety of the above-described results on human spatial working memory capacity in light of current models, which attempt to explain the apparent limits on the storage capacity of human visual working memory. On one hand, some researchers propose that working memory can be envisioned as being comprised of a limited number ( 3 or 4 ) of discrete "slots", each with all-or-none properties, that can store discrete, fixed-resolution representations, thus giving rise to a fixed-capacity model of working memory (Donkin, Nosofsky, Gold, \& Shiffrin, 2013; Luck \& Vogel, 1997a; Rouder et al., 2008). The latest version of the "slot model", the "slot + average model", suggests that an increase in memory precision for one item is possible when the stimulus set is lower than the limit and the same memory is stored in two slots (Zhang \& Luck, 2008). The "slot model" is consistent with classical views of working memory in which a limited number of items or chunks of information can be temporarily held in memory, and both Cowan's (Cowan, 2001) and Miller's (Miller, 1956) conceptions of working memory capacity fall into this category, with capacities limited to 4 and 7 , respectively. On the other hand, "resource models" propose that working memory comprises a pool of resources that can be allocated flexibly to provide either a small number of high-resolution representations or a large number of low-resolution representations (Bays \& Husain, 2008; Gorgoraptis, 
Catalao, Bays, \& Husain, 2011; van den Berg, Shin, Chou, George, \& Ma, 2012). These opposing theories have led researchers to conduct a myriad of studies on human visual working memory capacity, the results of which favor either the "slot model" or the "resource model", depending on the specifics of the experiment (Ma, Husain, \& Bays, 2014). However, the suitability of these models to account for allocentric spatial working memory capacity in the real world has not yet been evaluated.

Here, we aimed to determine the capacity of human short-term allocentric spatial memory in a real-world setting. Young adult men and women were tested on their ability to learn, on a trial-unique basis, and remember over a 1-min interval the location(s) of 1, 3, 5, or 7 illuminating pads, among 23 pads distributed in a real-world, $4 \mathrm{~m} \times 4 \mathrm{~m}$ arena surrounded by curtains on three sides. Participants had to walk to and touch the pads with their foot to illuminate the goal locations. We found that previous models of visual working memory could not account for the measures of allocentric spatial working memory capacity that we obtained in this real world paradigm. Instead, we found evidence that memories for individual locations are imprecise, and that memory performance for one location can be used to predict memory performance for multiple locations. Based on these data, we propose a theoretical model suggesting that memory precision determines the capacity of human short-term spatial memory.

\section{Materials and methods}

\subsection{Participants}

Participants were 42 undergraduate students from the Biomedical Sciences Program at the University of Fribourg, Switzerland (20 males, 22 females, age range 20.58-32.21 years, average 23.18 years). Subjects were tested for approximately 45 min each on four separate days, between 7 A.M. and 8 P.M. Human subjects research was approved by the Intercantonal Ethics Committee for Jura, Neuchatel, Fribourg (Neuchatel, Switzerland), and was in accordance with the NIH guidelines for the use of human subjects in research. All participants gave written, informed consent prior to participation.

A

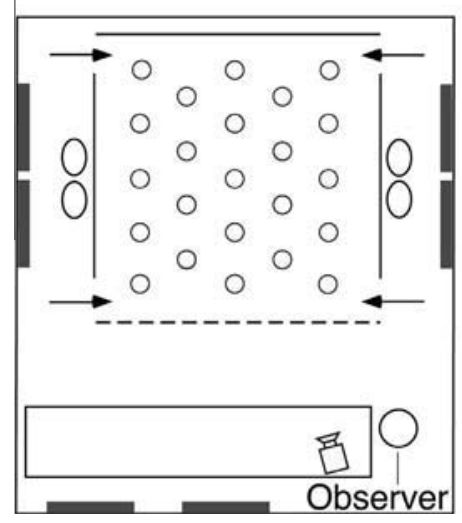

B

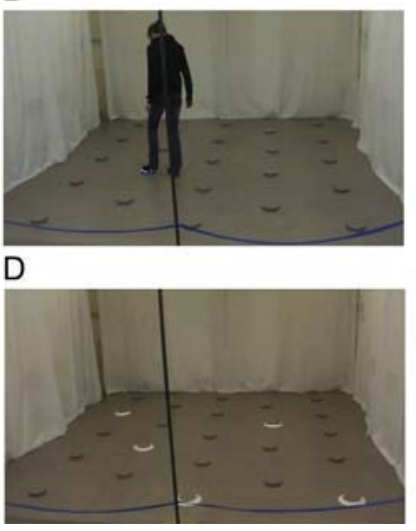

C

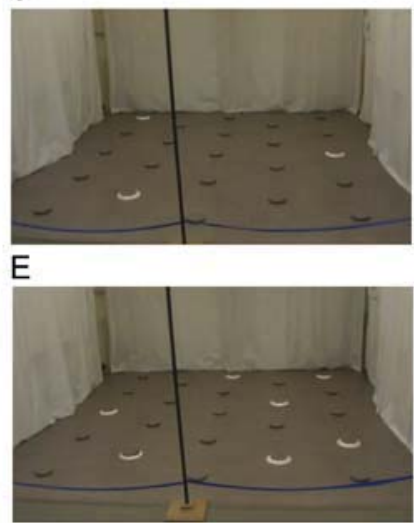

Fig. 1. Testing environment. (A) Schematic, aerial view of the experimental room $(6 \mathrm{~m} \times 7 \mathrm{~m})$ containing polarizing features such as doors, tables (white rectangle), chairs, and wall posters. Plastic curtains (solid lines) defined three of the boundary walls designating the arena. At each of the four near and far corners of the side walls was a $50 \mathrm{~cm}$ gap that served as one of the four different entry points (arrows) through which the subjects must pass in order to enter and exit the testing arena. Twenty-three foot pads, separated by $80 \mathrm{~cm}$ from each other, were regularly arranged in the arena. (B) Picture of the arena with a participant touching an illuminating pad. (C) Picture of the arena with three cued goal locations. (D) Picture of the arena with five cued goal locations. (E) Picture of the arena with seven cued goal locations. 


\subsection{Testing facility}

Testing took place in a large room $(6 \mathrm{~m} \times 7 \mathrm{~m}$; Fig. 1) containing many polarizing features such as doors, tables, chairs, cabinets, and wall posters. Within the room was a $4 \mathrm{~m} \times 4 \mathrm{~m}$ arena that consisted of 3 walls made of opaque plastic curtains ( $2 \mathrm{~m}$ high). Whereas the curtain on the back wall was $4 \mathrm{~m}$ wide, the curtains on the side walls extended only $3 \mathrm{~m}$, so that there was a $50 \mathrm{~cm}$ gap at the front and the back of the wall, thus creating four entry points through which participants passed in order to enter and exit the arena. Each entrance was marked by a number. The fourth (front) boundary of the arena was delineated by a rope attached to the two opposing side walls of the arena, and suspended $30 \mathrm{~cm}$ off the ground. Exterior to the two side walls, the inter-trial waiting area was a corridor $(1 \mathrm{~m} \times 4 \mathrm{~m})$ that contained two chairs and various items including a trash can, a door on one side, posters, etc., none of which could be viewed from within the arena. When subjects were not in the arena (i.e., during the inter-phase or inter-trial intervals), they sat in one of the chairs behind one of the two side walls with their back facing the arena. Importantly, from within the arena, and from the waiting area, participants had access to distant visual cues within the room.

The arena was empty except for 23 identical, regularly arranged gray foot pads (i.e., plastic disks, $15 \mathrm{~cm}$ in diameter and $2 \mathrm{~cm}$ high). The arena's floor was uniform, and thus could not provide participants with any local landmarks. Each foot pad was equipped with six L.E.D. lights arranged in a circle on the top outer edge of the disk. Pads designated as goal locations by the experimenter would illuminate when touched lightly with the foot, but the light would extinguish as soon as the foot was removed from the pad. Each goal location was chosen pseudorandomly, so that each particular location (1-23) served as goal location an average of $7 \pm 1$ times across all testing sessions. There was no overlap between configurations across the entire testing. Ten different locations were used for the first session of ten trials with ten trial-unique locations. Ten different configurations of three locations were used for the second session; no goal location could be directly adjacent to another goal location; any particular goal location could not be reused for at least two trials. Ten different configurations of five locations were used for the third session; in three trials (5, 8, and 10), two goal locations were immediately adjacent to each other; any particular goal location could not be reused for the next trial. Ten different configurations of seven locations were used for the fourth session; in seven trials $(1,3,4,5,6,7,9)$ two goal locations were immediately adjacent to each other; on five different occasions, one goal location was part of distinct configurations of rewarded locations on two successive trials (but not the same locations across occasions).

The design of this task allows us to assess allocentric, spatial relational memory (Banta Lavenex, Amaral, \& Lavenex, 2006; Banta Lavenex \& Lavenex, 2009; Banta Lavenex et al., 2014), that is, the memory for spatial locations defined in relation to distant environmental cues (i.e., viewpointindependent, in a frame of reference centered on the objects constituting the environment), and which is therefore independent from the subject's own location or orientation (i.e., not centered on the subject's body position or egocentrically). Importantly, this task tests spatial learning and memory in a controlled, real-world environment in which subjects have access to all sensory information normally available when moving about in everyday life, in contrast to experiments carried out on a table top, on a computer screen or in virtual environments.

\subsection{Procedure}

Participants were tested on their ability to learn and remember the location(s) of illuminating pads, on a trial-unique basis. Participants completed four successive sessions with 1, 3, 5, and 7 goal locations on days 1, 2, 3 and 4, respectively; sessions were not counterbalanced. Each session consisted of ten trials, and each trial consisted of separate encoding and recall phases. Participants were divided into two groups with respect to the information that was available during the encoding phase: For one group (10 males, average age 23.29 years, range $21.52-26.18$ years; 10 females, average age 22.24 years, range $20.58-24.19$ years), visual cues (white disks) surrounded the goal locations. Thus, upon entering the arena, participants could see all of the goal locations, and could potentially form a visual, snapshot memory of these locations from that specific entry. Once entering the arena, subjects were required to walk to each of the cued goal locations and touch the pad with their foot 
to illuminate it. As soon as participants removed their foot from the pad, its lights would extinguish. This insured that participants would not only see the goal locations from a distance, but also have the opportunity to encode the location of each goal in a multimodal allocentric frame of reference that included distal visual cues within the room. For the second group (10 males, average age 22.99 years, range 21.57-24.09 years; 12 females, average age 24.02 years, range 20.69-32.21 years), no visual cues were associated with the goals. Thus, upon entering the arena, participants would discover a new goal location (or 3, 5, 7 locations for the other sessions) by systematically visiting each of the pads until the goal(s) were found. As soon as participants removed their foot from the pad, its lights would extinguish. Thus in this condition, they could not form a snapshot memory of the goal locations from any particular point within the arena. The aim for the participants in both groups was to encode the location of the goal(s) in order to find it/them again when they returned to the arena in the recall phase, after a 1-min interval.

As soon as a participant had located the last goal during an encoding phase, $s /$ he was instructed to exit the arena by a predetermined door that the experimenter called out. During the 1-min interval between the encoding and recall phases of each trial, participants were required to count backward by 3 from a predetermined number (1000 in trial 1, 899 in trial 2, 798 in trial 3, 697 in trial 4, 596 in trial 5, 495 in trial 6, 394 in trial 7, 999 in trial 8, 898 in trial 9, and 797 in trial 10; The backward counting procedure was the same for the four sessions, which were always performed on separate days). Although the experimenter did not control counting accuracy, she listened for fluency and prompted participants to continue counting if any pauses were evident.

In order to impede the use of egocentric spatial representations, i.e., retracing their path through the arena, or seeing the same view that they had previously at the beginning of the encoding phase, participants were instructed to enter and exit the arena from different pseudorandomly chosen doors during encoding and recall phases. A 1-to-2-min intertrial interval (between the last recall phase and the encoding phase of the next trial) allowed the experimenter to rearrange the illuminating disks into the next trial-unique array. The same procedure repeated as described above for a total of ten trials within a daily session.

\subsection{Data analysis}

The following measures were used to analyze the subjects' performance and memory capacity: (1) the total number of locations visited to find the goal location(s) (TNV), as an overall measure of memory performance; (2) the number of goal locations visited before making an error (visiting a non-goal location; CBE), as a proxy to estimate memory capacity; (3) the number of errorless trials (NET), as a measure of perfect memory performance; (4) the number of trials in which the first location visited was a goal location (FCC), as a measure of memory performance for one location across memory loads; (5) the types of locations visited when subjects made an error (i.e., previous goal location, location adjacent to a goal, or other locations), as a measure of between-trial interference and within-trial spatial memory precision. We used General Linear Model analyses (GLMs) to compare performance of men and women in different testing conditions (cue vs no cue) and between different memory loads $(1,3,5,7$ locations). Since only marginal differences were observed for women tested in different encoding conditions (cue vs no cue) with memory loads of 1 and 7 , we pooled the data for males and females across testing conditions in order to simplify the presentation and analysis of the results regarding the influence of memory load on performance. For example, for TNV, we found no effect of sex $\left(F_{(1,38)}=0.255, p=0.616\right)$ or encoding condition $\left(F_{(1,38)}=0.883, p=0.353\right)$, but an interaction between sex and encoding condition $\left(F_{(1,38)}=4.573, p=0.039\right)$. Women had fewer TNV during the recall phase when a visual cue marked the goal location $(s)$ during encoding $\left(F_{(1,20)}=4.822\right.$, $p=0.040$; one location, $p=0.0125$; three locations, $p=0.8571$; five locations, $p=0.6336$; seven locations, $p=0.0687$ ). In contrast, men's TNV was not affected by the presence of local visual cue(s) marking the goal location(s) during encoding $\left(F_{(1,18)}=0.710, p=0.410\right)$. The other measures (CBE, NET, FCC) gave similar results (data not shown). Statistical significance was set at $p<0.05$ for all analyses.

In the Results, in addition to presenting each analyzed measure, we describe the results that would be predicted if allocentric spatial memory capacity was governed by the rules underlying classical slot models or resource models of working memory capacity. 


\section{Results}

\subsection{Total number of locations visited (TNV)}

We first considered the total number of locations visited to find the goal(s) for each memory load ("total number of visits", Fig. 2A), as an overall measure of memory performance. Recall that, as described above, slots in slot models are considered as all-or-nothing: either an item is placed into a slot and it is remembered, or all slots are full and the item is not remembered. Accordingly, Miller's magical number 7 predicts that TNV would be equal to the number of goals up to seven locations. Cowan's magical number 4 predicts that TNV would be equal to the number of goals for one and three goals, and would be determined by a random search of the goal(s) beyond four, following the formula: $\mathrm{TNV}=4+n *(x+1) /(n+1)$, where $n$ is the number of goals above four and $x$ is the number of remaining unvisited locations. Thus, for five locations, TNV $=4+1 *(19+1) /(1+1)=14$; for seven locations, $\mathrm{TNV}=4+3 *(19+1) /(3+1)=19$. In contrast, we found that TNV increased linearly with the number of goals from one to seven locations $\left(F_{(3,123)}=292.363, p<0.001\right.$; TNV $=0.21+1.55 *$ goals $)$. This finding is inconsistent with basic slot models of working memory capacity (such as Cohen's and Miller's magical numbers) that do not predict a linear increase in TNV with increasing memory load.

To enable statistical comparison of memory performance between different memory loads, TNV was normalized by dividing it by the number of goals for each memory load (i.e., 1, 3, 5 or 7; Fig. 2B). Normalized TNV did not vary as a function of memory load $\left(F_{(3,123)}=0.395, p=0.757\right.$; $\mathrm{TNVn}=1.64-0.01 *$ goals $)$, and was about 1.6 for all memory loads. This finding is inconsistent with the slot + average model and the resource model of working memory performance, which would predict that the precision of memory representations should decrease with increasing memory loads, regardless of whether there is an upper limit. If memory precision decreased with increasing memory load, relative TNV should increase, unless the precision of the memory representation for each of the seven goal locations remains sufficient to distinguish these locations from adjacent locations $80 \mathrm{~cm}$ away.

\subsection{Number of correct choices before the first error ( $C B E)$}

We then considered the number of goal locations subjects visited before making an error ("correct before error"; Fig. 3A). This measure represents a proxy to estimate memory capacity. Miller's magical number 7 predicts that CBE would be equal to the number of goals up to seven locations. Cowan's magical number 4 predicts that $\mathrm{CBE}$ would be equal to the number of goals for one and three goals,
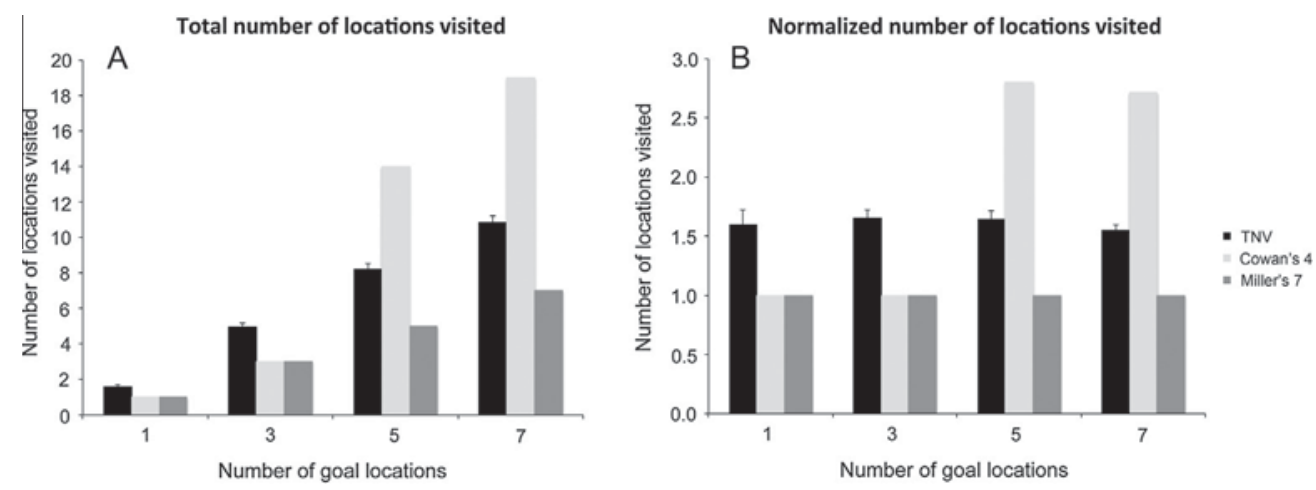

Fig. 2. Total number of locations visited (TNV) to find the goal location(s). (A) Total number of locations visited per trial. (B) Normalized number of locations visited per trial: TNV divided by the number of goal locations. Black bars: actual performance (average for all participants \pm SE); light gray bars: predictions based on Cowan's magical number 4; dark gray bars: predictions based on Miller's magical number 7. 

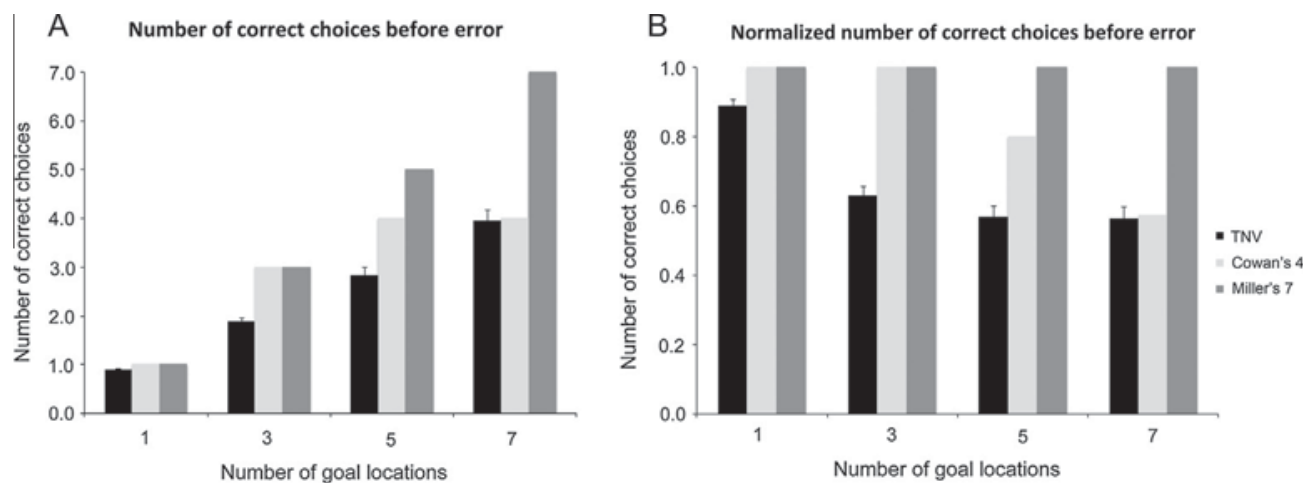

Fig. 3. Number of correct choices before making the first error (CBE). (A) Number of correct choices per trial. (B) Normalized number of correct choices: CBE divided by the number of goal locations. Black bars: actual performance (average for all participants $\pm \mathrm{SE}$ ); light gray bars: predictions based on Cowan's magical number 4; dark gray bars: predictions based on Miller's magical number 7.

and would be equal to four (the maximal memory capacity) for five and seven locations. In contrast, we found that $\mathrm{CBE}$ varied as a function of memory load $\left(F_{(3,123)}=111.102, p<0.001\right.$; $\mathrm{CBE}=0.37+0.50 *$ goals, from 1 to 7 goals), increasing from 0.89 for one location, to 1.90 for three locations, to 2.85 for five locations and to 3.99 for seven locations. Although CBE for seven locations is equal to an upper limit of 4 , our findings for three and five locations are too low to be consistent with Cowan's magical number 4 and basic slot models. In contrast, we determined that CBE could be predicted based on the proportion of correct first choices for one location (FCC, see also Section 3.4; $\mathrm{CBE}=\mathrm{FCC}_{1}+(X-1) / 2$, where $X=$ number of goals $)$.

Because CBE is limited by the number of goals, and in order to enable statistical comparison between memory loads, CBE was normalized by dividing it by the number of goals (Fig. 3B). Miller's magical number 7 predicts that normalized CBE would be equal to one for all memory loads; Cowan's magical number 4 predicts maximal performance for one and three goals ( $\mathrm{CBE}=1 / 1$ for $1 ; \mathrm{CBE}=3 / 3$ for 3 ) and an exponential decrease in performance from three to seven locations ( $C B E=4 / 5=0.80$ for 5 ; $C B E=4 / 7=0.57$ for 7 ); resource models predict a linear decrease in performance from one to seven locations. In contrast, although we found that the normalized CBE decreased with increasing memory load $\left(F_{(3,123)}=53.419, p<0.0001: 1\right.$ location $>3$ locations $>5$ locations $=7$ locations $)$, there was a large decrease in performance from one $(\mathrm{CBE}=0.89)$ to three $(\mathrm{CBE}=0.63)$ locations, and no significant difference between five $(\mathrm{CBE}=0.57)$ and seven $(\mathrm{CBE}=0.56)$ locations. These findings are thus inconsistent with slot and resource models of working memory performance.

\subsection{Number of errorless trials (NET)}

We further considered the number of trials in which subjects made no error ("number of errorless trials"; Fig. 4A), as a measure of perfect memory performance. Miller's magical number 7 predicts that NET would be equal to the number of trials $(n=10)$ for each memory load up to seven locations. Cowan's magical number 4 predicts that CBE would be equal to the number of trials $(n=10)$ for one and three goals, and would be equal to zero for five and seven locations, since no participant should be able to remember more than four locations on any given trial. With a slightly looser definition of memory limits to $4 \pm 1$, one would predict that a proportion of trials might be performed errorlessly with a memory load of five, but none for a memory load of seven. In contrast, we found that the number of errorless trials decreased non-linearly with memory load (NET $=14.05-6.06$ load +0.83 load $^{2}$; $F_{(3,123)}=98.934, p<0.0001 ; 1$ location $>3$ locations $>5$ locations $=7$ locations $)$. These findings are inconsistent with basic slot models of working memory performance.

To take into account the fact that part of the performance might be influenced by chance, we corrected the observed numbers of errorless trials by subtracting the probability to make errorless trials 

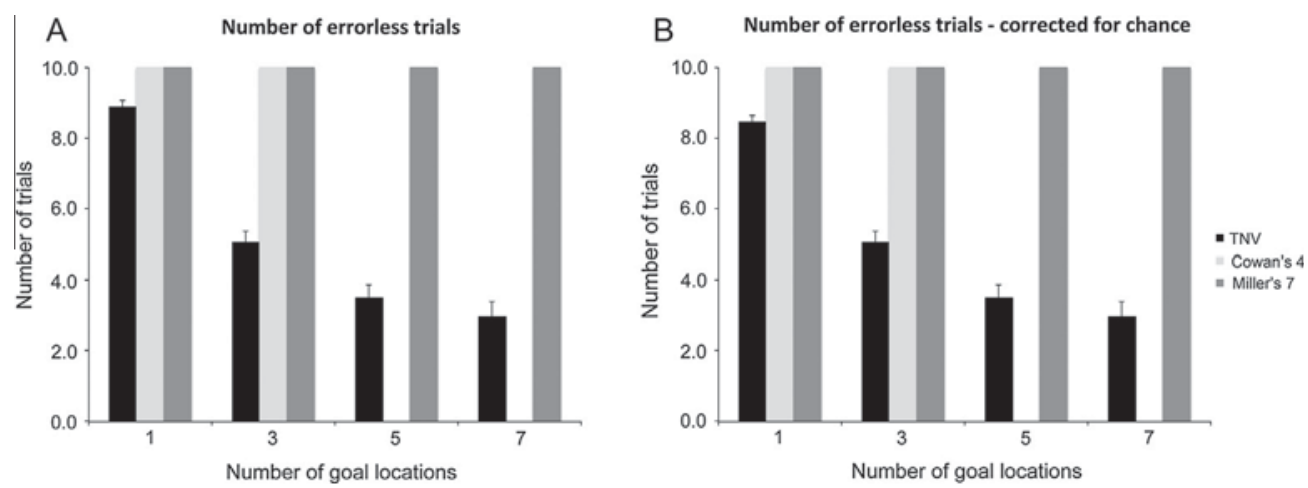

Fig. 4. Number of errorless trials (NET): Number of trials in which subjects made no errors. (A) Number of trials. (B) Number of trials corrected for the probability to find all goal locations by chance: 0.43478 with one location $(1 / 23 * 10) ; 0.00565$ with 3 locations $(3 / 23 * 2 / 22 * 1 / 21 * 10)$; 0.00030 with 5 locations $(5 / 23 * 4 / 22 * 3 / 21 * 2 / 20 * 1 / 19 * 10)$; and 0.00004 with 7 locations $(7 / 23 * 6 / 22 * 5 / 21 * 4 / 20 * 3 / 19 * 2 / 18 * 1 / 17 * 10)$. Black bars: actual performance (average for all participants \pm SE); light gray bars: predictions based on Cowan's magical number 4; dark gray bars: predictions based on Miller's magical number 7 .

by chance in the four memory load conditions (Fig. 4B): 0.43478 with one location $(1 / 23 * 10)$, 0.00565 with 3 locations $(3 / 23 * 2 / 22 * 1 / 21 * 10), 0.00030$ with 5 locations $(5 / 23 * 4 / 22 * 3 / 21 * 2$ / $20 * 1 / 19 * 10)$ and 0.00004 with 7 locations $(7 / 23 * 6 / 22 * 5 / 21 * 4 / 20 * 3 / 19 * 2 / 18 * 1 / 17 * 10)$. Note that this correction is relatively important for a memory load of one, but is essentially negligible for memory loads of three, five and seven. The corrected NET decreased with increasing memory load $\left(\right.$ corrNET $=13.08-5.40 \mathrm{load}+0.72 \operatorname{load}^{2} ; F_{(3,123)}=115.804, p<0.0001 ; 1$ location $>3$ locations $>5$ locations $=7$ locations). Again, we found a large decrease in NET from one to three locations and relatively smaller decreases from three to seven locations.

In sum, the number of errorless trials decreased with increasing memory load from 1 to 3 to 5 locations, but did not differ between 5 and 7 locations. These findings are inconsistent with basic slot models and resource models of working memory performance. In Section 3.8, we present a mathematical model that can predict the number of errorless trials with different memory loads, based on the performance with one location.

\subsection{Number of trials with the first choice correct (FCC)}

We analyzed the number of trials in which participants chose a goal location as their first choice upon entering the arena ("first choice correct"; Fig. 5A). Slot models predict that individuals should be able to remember at least one location accurately and therefore be correct in their first choice upon entering the arena on every trial $(n=10)$, irrespective of memory load (Note that even if performance is not perfect due to other factors influencing behavior, FCC should be the same for all memory loads, for example around $90 \%)$. In contrast, we found an effect of memory load $\left(F_{(3,123)}=6.159, p<0.001 ; 1\right.$ location $>3$ locations $=5$ locations; 7 locations $>3$ locations), reflecting what appeared to be a decrease of FCC from one $(F C C=8.9)$ to three $(F C C=7.8)$ locations, and a linear increase from three to five $(\mathrm{FCC}=8.2)$ to seven $(\mathrm{FCC}=8.6)$ locations ( $\mathrm{FCC}=7.28+0.19$ goals, from 3 to 7 goals $)$. An increase in FCC with increasing memory load is not consistent with any theoretical model and likely reflects the influence of chance on the choice of the first location upon entering the arena.

Thus, in order to compare the part of the performance that can be unambiguously attributed to memory, FCC was corrected by subtracting the probability of finding the first goal by chance with different memory loads (i.e., $1 / 23,3 / 23,5 / 23$ or $7 / 23$, respectively; Fig. 5B). The corrected FCC decreased with increasing memory load $\left(F_{(3,123)}=48.561, p<0.0001 ; 1\right.$ location $>3$ locations $>5$ locations $>7$ locations). As was observed for $\mathrm{CBE}$, we found a large decrease in corrected FCC from one $(F C C=8.5)$ to three $(F C C=6.5)$ locations, and a linear decrease from three to five $(F C C=6.0)$ to seven 

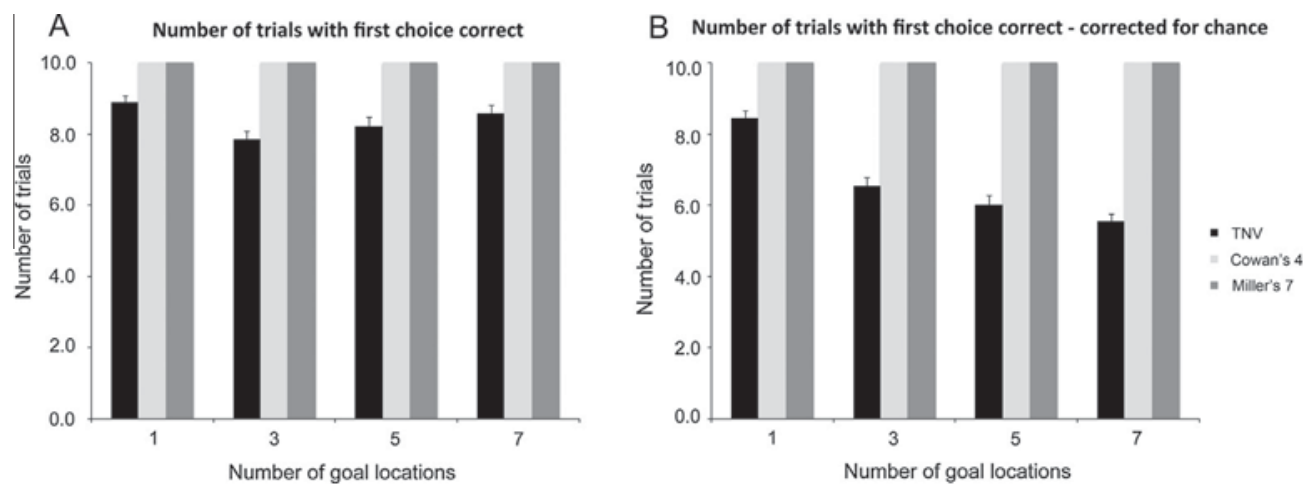

Fig. 5. Number of trials with the first choice correct (FCC). (A) Number of trials. (B) Number of trials corrected for the probability to find the first goal location by chance: $1 / 23$ for one location; $3 / 23$ for three locations; $5 / 23$ for five locations; $7 / 23$ for seven locations. Black bars: actual performance (average for all participants \pm SE); light gray bars: predictions based on Cowan's magical number 4; dark gray bars: predictions based on Miller's magical number 7 .

$(\mathrm{FCC}=5.5)$ locations ( FCC $=7.28-0.25$ goals, from 3 to 7 goals). Note that the difference in the slopes of the linear relationships reported for the raw and normalized FCC reflects the correction for chance $(-1 / 23$ per goal $\times 10$ trials $)$.

In sum, the interpretation of FCC analyses may vary based on the assumptions one makes regarding the impact of chance on behavior. Assuming that chance plays no role, one would conclude that FCC increases with the number of goals when memory loads higher than one are compared, i.e., from three, to five, to seven. This finding would be rather counter-intuitive and inconsistent with slot and resource models. In contrast, when considering the part of the performance that can be unambiguously attributed to memory, one would conclude that FCC decreases with increasing memory load. This finding would be consistent with resource models. Interestingly, considering only a partial influence of chance on behavior (arbitrarily chosen at $42 \%$ of the effect of chance) results in a large decrease in FCC from one $(\mathrm{FCC}=8.7)$ to three $(\mathrm{FCC}=7.3)$ locations and a stable performance from three to five to seven locations (FCC $=7.3$ ). These analyses highlight the difficulty in interpreting experimental findings, which may be considered consistent or inconsistent with different models depending on the specific experimental parameters and a priori assumptions regarding the influence of chance on performance.

\subsection{Spatial memory precision}

In order to characterize sub-optimal memory performance, we analyzed the types of locations chosen when participants made an error in their first choice upon entering the arena. Specifically, we analyzed whether incorrect first choices made with a memory load of one, or three, corresponded to: (1) the previous goal location(s), thus representing a difficulty in distinguishing between different trials (i.e., a temporal resolution error); (2) locations adjacent to the goal location(s), thus representing a difficulty in distinguishing closely apposed locations (i.e., a spatial resolution error); (3) other unrelated, random locations. Note that the numbers of different types of choices were normalized based on the probability to make those choices (i.e., the number of locations in this category). We could not perform this analysis for five and seven goal locations since an incorrect choice often belonged to two different categories, i.e., a previous location was also an adjacent location, and incorrect locations could be adjacent to more than one goal location.

We found that the subjects' choices were not influenced by the goal location(s) of the previous trial, i.e. there was no between-trial interference. With a memory load of one, a previous location was chosen only once out of a total of 42 errors made by 25 subjects (for trials 2-10). However, when subjects made an error in their first choice upon entering the arena, their choice was not random; instead, they chose more often locations adjacent to the goal than other locations (Adjacent: $0.100 \pm 0.021$, Other: $\left.0.033 \pm 0.005 ; t_{(24)}=2.674 ; p=0.01328\right)$. With a memory load of three, subjects also chose more 
locations adjacent to one of the goals than other locations (Adjacent: $0.074 \pm 0.006$, Other: $0.038 \pm 0.009 ; t_{(31)}=2.387 ; p=0.02317$; no previous location was chosen).

In sum, when participants made an error, they chose locations adjacent to the goal(s). This suggests that although their memory was not perfect, they remembered the area where the goals were located: their memory was accurate, but not precise.

\subsection{Number of trials with $n$ correct choices (NCC)}

In addition to the number of correct choices before erring (CBE) and the number of first choices correct (FCC), we evaluated the number of trials in which $n$ consecutive correct choices were made (NCC; i.e., the first choice was correct, the first two choices were correct, the first three choices were correct, etc.; Fig. 6A). We found that NCC followed a different linear relationship for each memory load: three locations, $\mathrm{NCC}=9.13-1.43 *$ goals; five locations, $\mathrm{NCC}=9.20-1.18 *$ goals; seven locations, NCC $=9.46-0.96 *$ goals. Note that in Fig. $6 \mathrm{~A}$, the values for one correct choice correspond to the number of trials with the first choice correct (FCC; Fig. 5A); the values for $100 \%$ correct choices correspond to the number of errorless trials (NET; Fig. 4A).

The different regression lines for different memory loads are likely due to the influence of chance on performance. Most importantly, however, the number of trials with the first choice correct observed for each memory load $\left(\mathrm{FCC}_{x}\right)$ can be used to predict the number of correct choices before erring: $\mathrm{CBEpre}_{x}=X * \mathrm{FCC}_{x}^{((X+1) / 2)} ; X$ is the number of locations to remember (Fig. 6B). These findings thus further suggest that memory capacity is related to the precision of the memories for individual locations.

\subsection{Organization of encoding and retrieval paths}

In addition to the analyses of correct choices and errors described above, we evaluated whether performance was related to the organization of the subjects' encoding and retrieval paths. Specifically, for a memory load of seven, we calculated a score reflecting the subjects' tendency to visit the different goal locations following the same sequence (either forward or backward, i.e., in the reverse order) during the recall phase as during the encoding phase. If, during the encoding phase, a subject visited the goal locations in a given order (for example locations $20,17,12,10,7,5,1$ ), during recall one point would be allotted for every sequence of two locations visited in the same or reverse order as during encoding (for example, 20-17, or 5-7). Thus, during the recall phase, the sequence " $1,20,17,12$, $10,7,5$ " would obtain a score of 5 , and the series " $17,20,10,5,12,7,1$ " would obtain a score of 1 (the maximum score for 7 locations being 6 ). We found statistically significant correlations between sequential search paths and task performance, which differed between males and females depending
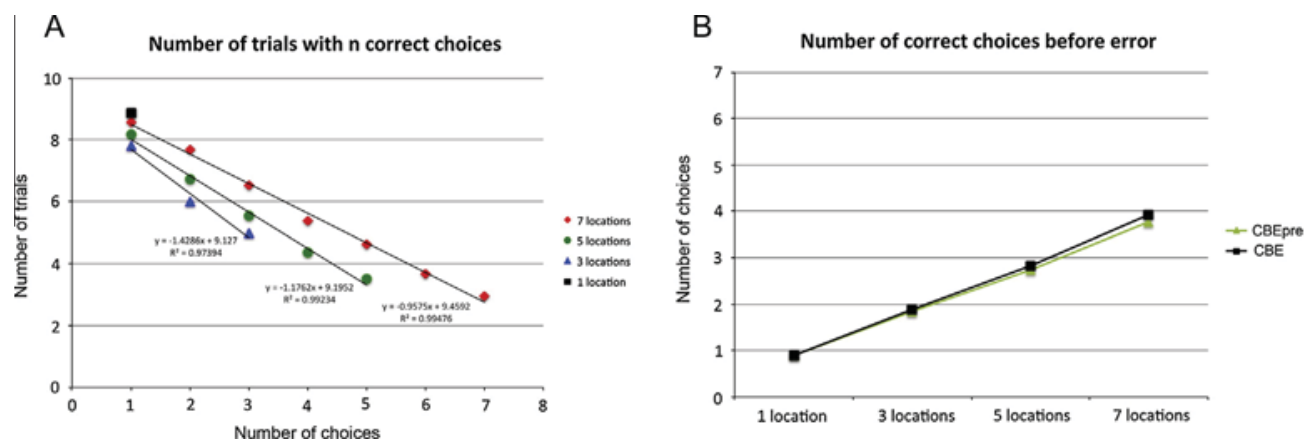

Fig. 6. Number of correct choices before error, detailed analyses. (A) Number of trials with $n$ correct choices (NCC). (B) Number of correct choices before error. Black squares: observed (CBE); green triangles: predicted numbers $(\mathrm{CBEpre})$. $\mathrm{CBEpre}{ }_{x}=$ $X * \mathrm{FCC}_{X}((X+1) / 2)$. See main text for details. (For interpretation of the references to color in this figure legend, the reader is referred to the web version of this article.) 
on whether local cues marked the goal locations or not. When local cues marked the goal locations during encoding, females' total number of locations visited (TNV) during retrieval decreased as their sequential search score (SSS) increased (Fig. 7A; TNV $=20.24-0.25 * \mathrm{SSS}, p=0.021, R^{2}=0.507$ ); males did not exhibit such a correlation (TNV $=12.11-0.05 *$ SSS, $p=0.625, R^{2}=0.031$ ). In contrast, when no local cues marked the goal locations during encoding, males' TNV during retrieval decreased as their SSS increased (Fig. 7B; TNV $=19.78-0.22 * \mathrm{SSS}, p=0.004, R^{2}=0.638$ ); whereas females did not exhibit such a correlation (TNV $\left.=16.24-0.10 * \mathrm{SSS}, p=0.161, R^{2}=0.156\right)$. Note, however, that the vast majority of subjects did not follow strict sequential paths, and that the sequential search score considered sequential visits either forward or backward, so that a high score does not necessarily imply that subjects recapitulated the exact same path during encoding and recall phases.

In sum, we found that sequential searching correlated with recall performance for seven locations. However, whether search paths influenced memory performance or memory performance influenced search paths remains to be determined.

\subsection{Modeling memory capacity for multiple items}

Based on our results described above, we developed a model to predict the number of accurate complex memories one could form and retrieve based on the ability to remember one location. First, we determined the proportion of errorless trials with different memory loads (Table 1A). Second, we corrected the observed proportion of errorless trials based on the probability to make an errorless trial by chance (Table $1 \mathrm{~A} ; 0.043478$ with one location (1/23), 0.000564 with 3 locations $(3 / 23 * 2 / 22 * 1 /$ 21), 0.000029 with 5 locations $(5 / 23 * 4 / 22 * 3 / 21 * 2 / 20 * 1 / 19)$ and 0.000004 with 7 locations (7/ $23 * 6 / 22 * 5 / 21 * 4 / 20 * 3 / 19 * 2 / 18 * 1 / 17)$ ). Third, we inferred the number of locations that could be remembered as a function of the corrected proportion of errorless trials and the number of goals (Table 1B). For example, we observed a proportion of 0.505 errorless trials for three goals; following the correction for finding the three goals by chance $(-0.000564)$, the corrected proportion of errorless trials was 0.504 . Considering that there were three goal locations, one could extrapolate that on average 1.513 locations were remembered accurately (Table $1 \mathrm{~B} ; 3 \times 0.504$ ). This is a theoretical inference derived from our experimental data designed to estimate the number of locations that can be remembered accurately. Finally, we compared this inferred number to the predicted number of locations that can be remembered accurately based on the proportion of errorless trials with one location. In our case, we found a corrected, observed proportion of 0.845 errorless trials with one location (0.8880.043 ), which corresponds precisely to 0.845 locations. If the subjects' memory capacity for three locations was a direct function of the memory performance for one location, $P_{x}=\left(P_{1 \mathrm{Cor}}\right)^{X}$, we would predict that a total of 1.810 locations should be remembered accurately $(0.845 * 0.845 * 0.845 * 3)$. This predicted value (1.810) is about $20 \%$ higher than the calculated value derived from our
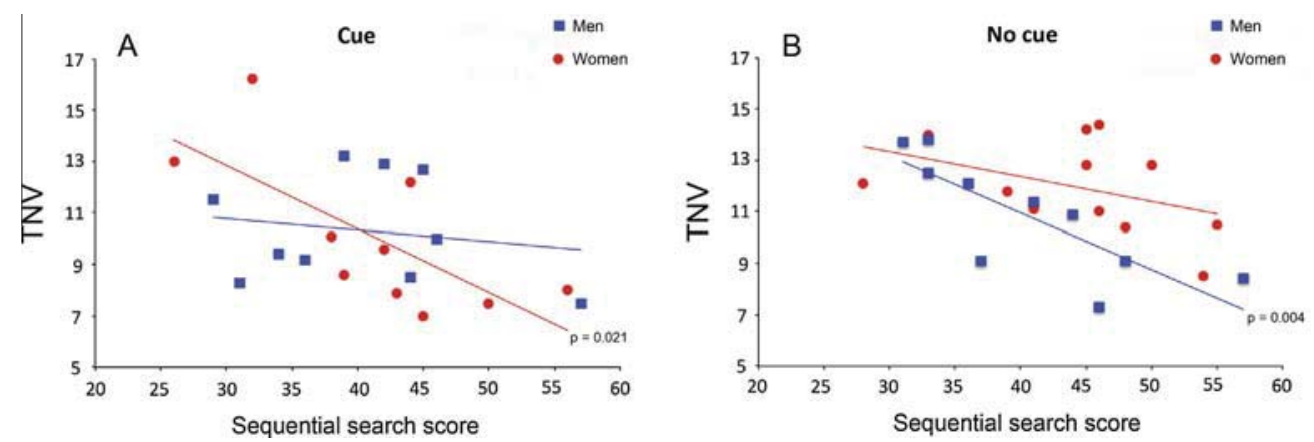

Fig. 7. Effect of the organization of encoding and retrieval search paths on task performance with seven goal locations Regression analyses between the total number of visits (TNV) and the sequential search score (SSS). (A). Performance when local cues marked the goal locations during encoding. Females: TNV $=20.24-0.25 \mathrm{SSS}, p=0.021, R^{2}=0.507$. (B) Performance when no local cues marked the goal locations during encoding. Males: TNV $=19.78-0.22$ SSS, $p=0.004, R^{2}=0.638$. 
A Performance for one location predicts memory capacity

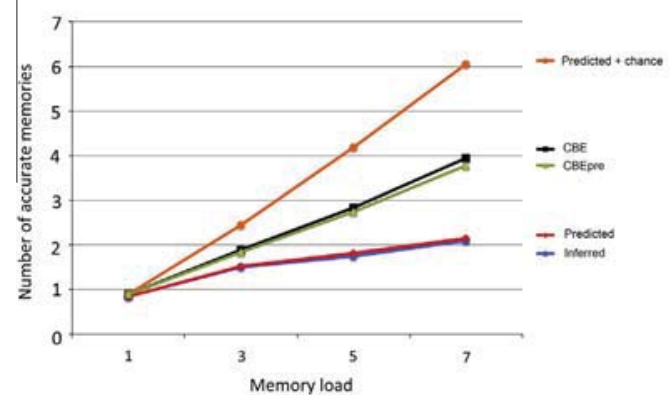

B Memory precision predicts memory capacity

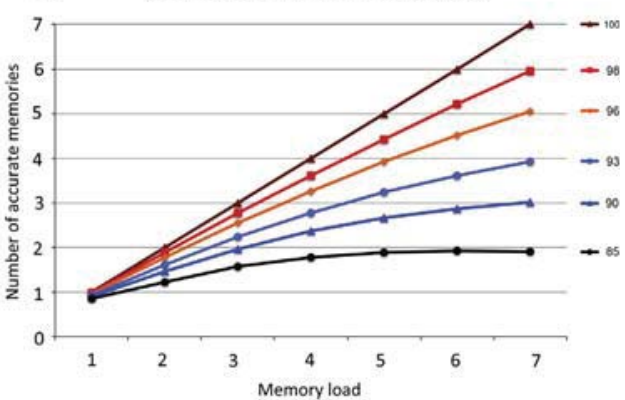

Fig. 8. Modeling memory capacity for multiple locations. (A) Number of accurate memories determined by (1) the number of correct choices before error $(\mathrm{CBE})$; $(2)$ the proportion of first correct choices with the same memory load $\left(\mathrm{FCC}_{x}\right)$ : $\mathrm{CBEpre}_{x}=$ $X * \mathrm{FCC}_{X}^{((X+1) / 2)}$; (3) memory performance for one item (Predicted). $P_{X}=\left(P_{1 \mathrm{Cor}}\right)^{(X+1)}$. Note that $X+1$ is limited to seven, so that $X+1=4$ for three locations, $X+1=6$ for 5 locations and $X+1=7$ for seven locations; (4) the number of errorless trials (Inferred); (5) memory performance for one location and the influence of chance (Predicted + chance). (B). Number of accurate memories predicted based on the memory performance for one location. $P_{X}=\left(P_{1 \mathrm{Cor}}\right)^{(X+1)}$. Note that the value of $X+1$ is not limited here, so that $X+1=4$ for three locations, $X+1=6$ for 5 locations and $X+1=8$ for seven locations. Small variations in memory precision (from $85 \%$ to $93 \%$ ) have a very strong influence on the number of accurate memories that can be remembered. See main text for details.

in memory precision (evaluated by the proportion of errorless trials with one location, or the proportion of first choice correct with several locations) can have a very significant impact on the number of locations that can be remembered accurately (Table 1 ; Fig. 8). If we compute the predicted numbers of remembered locations, without excluding chance (Table 1D), by adding the probability to find the goal location by chance to the corrected proportion of errorless trials for one location (e.g., for the first choice with a memory load of $3: 3 / 23+0.845=0.9754$; note that this sum is necessarily limited to $\leqslant 1$ ), we find that the numbers of remembered locations are even greater than those derived from the observed numbers of correct choices before error (CBE; Table 1E, Fig. 8A).

In sum, we determined that with a less than perfect memory $(<100 \%$ retrieval performance for one location) the proportion of accurate memories decreases as memory load increases. With a memory performance of $85 \%$ for one location, we predicted that although the proportion of remembered locations decreases with increasing memory loads, the total number of accurate memories increases significantly from 1 to 3 , to 5 , to 7 locations; this prediction was confirmed by our experimental data.

\section{Discussion}

In his seminal work published in 1956, George Miller described the magical number $7 \pm 2$, as the limit of our capacity for processing information and immediate memory capacity (Miller, 1956). A more recent view advocated by Nelson Cowan considers that mental storage capacity is more limited, and describes the magical number $4 \pm 1$ as the limit for pure short-term memory capacity in humans (Cowan, 2001). These views have led to current proposals that working memory stores a limited set (3 or 4) of discrete, fixed-resolution representations, also known as discrete "slots", each with all-ornone properties, which results in a discrete fixed-capacity model of working memory (Donkin et al., 2013; Luck \& Vogel, 1997b; Rouder et al., 2008; Zhang \& Luck, 2008). Our experimental results do not support the idea of a fixed, upper limit of 4 or 7 for short-term allocentric spatial memory capacity in humans. Our findings are also inconsistent with the proposal that working memory consists of a pool of resources that can be allocated flexibly to provide either a small number of high-resolution representations or a large number of low-resolution representations (Bays \& Husain, 2008; Gorgoraptis et al., 2011; van den Berg et al., 2012). Instead, we determined that the number of locations held in short-term allocentric spatial memory can be predicted based on memory performance for individual locations. Our findings are consistent with a theoretical model suggesting that 
the precision of the memory for individual locations might determine the capacity of human shortterm memory for spatial information.

\subsection{Comparison with previous studies}

We found that short-term spatial memory capacity varied as a function of memory load. Specifically, we found an average memory capacity (measured by the number of correct choices before error: CBE) of 0.89 for one location, 1.90 for three locations, 2.85 for five locations and 3.99 for seven locations. These findings are inconsistent with slot models of working memory that suggest that memory capacity is fixed regardless of the number of items to remember. If Miller's theory of memory capacity being limited to $7 \pm 2$ is accurate for allocentric spatial memory, then we should have observed a memory capacity of one for one location, three for three locations, five for five locations, and seven for seven locations. If Cohen's theory of memory capacity being limited to $4 \pm 1$ is accurate for allocentric spatial memory, then we should have observed one for one location, three for three locations, and four (or 5) for five and seven locations. Obviously, Miller's and Cohen's estimates of working memory capacity do not hold for estimates of allocentric spatial working memory as assessed with the current paradigm.

Our current estimates of short-term spatial memory capacity could not be predicted based on previous studies of spatial memory capacity using the Corsi Block-tapping Task (CBT; (Corsi, 1972; Piccardi et al., 2013)), the Walking Corsi Test (WalCT; (Piccardi et al., 2013)) or the Memory game (Banta Lavenex et al., 2011). In his original description of the CBT, Corsi found that the immediate, sequential spatial memory span was 4.9 for normal controls (Corsi, 1972). Replications of the CBT revealed an average spatial memory span around $6 \pm 1$ for normal controls (Farrell Pagulayan et al. 2006). Normative data in healthy men and women, $15-25$ to 69-86 years of age (Piccardi et al., 2013), revealed serial-spatial memory spans for immediate recall ranging from $4.09 \pm 0.85$ in older women to $5.96 \pm 0.64$ in younger men. Serial-spatial memory spans for delayed recall ( 5 min delay) ranged from $5.04 \pm 2.67$ in older women to $7.37 \pm 1.54$ in younger women. In the WalCT, serial-spatial memory spans for immediate recall ranged from $3.65 \pm 0.83$ in older women to $5.61 \pm 1.04$ in younger men; serial-spatial memory spans for delayed recall ( 5 min delay) ranged from $6.26 \pm 2.43$ in older women to $7.93 \pm 0.36$ in younger women. Using the Memory game, we previously found that with 8 pairs of images (16 locations), individuals remembered an average of 3.33 items in egocentric conditions and 2.75 in allocentric conditions; with 12 pairs of images (24 locations), they remembered an average of 3.91 items in egocentric conditions and 2.99 in allocentric conditions (Banta Lavenex et al., 2011).

The large and paradigm-specific variability in the estimates of human spatial memory capacity described above (average: 4.9; range: $2.75-7.37$ ) precludes current slot models as viable theoretical constructs to explain short-term allocentric spatial memory. Specifically, slot models do not predict that memory capacity would be less than the number of items to be remembered, when the number of items to be remembered is inferior to the hypothesized limit of memory storage. Nor do they predict that memory capacity might increase with increasing memory load while the number of items to remember remains below the limit. Similarly, resource models of visual working memory do not account for our current findings and the wide range of estimates of human short-term spatial memory reported previously. Specifically, none of the measures of memory performance reported here (TNV CBE, NET, FCC, NCC) are consistent with an equal representation of all items to be remembered, accompanied by a decrease in memory precision with increasing memory load.

Instead, we found that the number of correct choices before erring (CBE), a measure used to estimate memory capacity in previous studies, could be predicted based on the participants' performance for their first choice upon entering the arena with a given memory load $(X): \operatorname{CBEpre}_{X}=X * \mathrm{FCC}_{X}^{((X+1) / 2)}$. Similarly, the number of errorless trials with a number of locations, $X$, could be predicted based on the number of errorless trials for one location: $P_{x}=\left(P_{1 \mathrm{Cor}}\right)^{(X+1)}$. Interestingly, detailed analyses of errors with one or three locations to be remembered revealed that when participants made an error, they chose locations adjacent to the goal location(s). Thus, although their memory was not perfect, they remembered the area where the goals were located: their memory was accurate, but not precise. 
Consequently, we propose that human short-term spatial memory capacity is determined by the precision of memories for individual locations, i.e., the degree of spatial resolution that is encoded. In our experiment, the existence of long-term memories for the locations to be remembered (i.e., reference memory for the array of 23 locations) and the opportunity to perceive all potential locations concurrently might explain why the precision of memories for individual locations does not decrease with increasing memory loads. Since the different sessions with different memory loads were not counterbalanced, it is possible that practice might have influenced performance in higher memory load conditions that were performed later in the sequence. However, we believe that practice is unlikely to have had a differential impact on performance, from 3 to 7 locations, since participants had ample opportunity to form a mental representation of all locations, which moreover remained visible throughout testing. We now consider the results of previous studies in light of our hypothesis that memory precision limits short-term spatial memory capacity. In the final section, we briefly discuss a neurobiological perspective that supports this hypothesis.

\subsection{Precision predicts capacity}

Miller's article on the magical number seven is often referred to in order to describe the limit of immediate memory capacity, yet his article was primarily focused on how accurately people can assign numbers to the magnitudes of various aspects of a stimulus, that is the ability to make absolute judgements. He described these experiments as investigations on the capacity of people to transmit information. Discussing these concepts in terms of information theory led him to consider that the span of immediate memory should vary as a function of the amount of information necessary to represent each item. We believe that, indeed, the precision with which the brain encodes information impacts similarly both the capacity to make absolute judgements and the span of immediate memory. We first review the experiments on absolute judgements described by Miller, considering the coding precision of neuronal assemblies within different sensory modalities. We then extend our discussion to experiments on memory capacity.

\subsubsection{Absolute judgements}

The first experiment discussed by Miller was on people's ability to make absolute judgements of unidimensional stimuli (i.e., stimuli that vary in only one dimension), such as tones. He reported the results of experiments showing that when subjects are asked to distinguish different tones (sounds varying in frequencies), they were usually unable to assign them to six different classes without error. When asked to make decisions based on loudness, subjects were able to distinguish only five perfectly discriminable alternatives. Miller concluded that the ability to make absolute judgements based on pitch or loudness were of the same order of magnitude, yet he also noted that absolute judgements of pitch were slightly more accurate than absolute judgements of loudness. Absolute judgements for taste intensities (different concentrations of a salt solution) revealed that subjects were capable of judging about four distinct concentrations. In contrast, the capacity for judgements of visual position seemed to be larger with about ten positions along a linear interval (note that chunking of information, left vs right, might have contributed to better performance in this task). Absolute judgements for touch, measured by placing vibrators on the chest region of test subjects, revealed that a good observer could identify about four intensities, about five durations, and about seven locations. Miller concluded by saying that: "There seems to be some limitation built into us either by learning or by the design of our nervous systems, a limit that keeps our channel capacities in this general range". We agree with this statement and would like to further propose that the precision of the neural coding specific to the nature of the stimuli in different sensory modalities might explain the differences in our ability to make absolute judgements. For example, within the auditory system, each auditory receptor responds optimally to a minimal amplitude stimulation of a given frequency, based on its location along the basilar membrane. Increasing sound amplitude for the same frequency increases the firing rate of this receptor and recruits receptors located nearby. In contrast, a second stimulus with a different frequency recruits receptors located elsewhere along the membrane. Accordingly, auditory stimuli varying in frequency (different tones) are encoded more distinctively by our nervous system than stimuli varying in amplitude (different loudness). Less overlap between the neural codes (i.e., 
ensembles of active neurons) representing these tones enables a more precise representation of the different stimuli and thus facilitates discriminative decisions. Similarly, differences in salt concentration are encoded by different firing rates of the same population of neurons coding for salt, and thus enable a less precise discrimination than a taste discrimination based on different tastes (salt, bitter, acid, sweet, umami), which recruit different neuronal populations. For touch perception, the precision of the neural coding for different properties of sensory stimuli can also explain why one can distinguish a larger number of stimuli based on their location, than based on their duration or intensity. The behavioral results described by Miller are thus consistent with the view that the precision in the neural coding of information at the sensory and perceptual levels might contribute to the variations in our abilities to make absolute judgements across different dimensions of stimuli in different sensory modalities. This view is consistent with the ideal observer model of visual working memory proposed by Sims and colleagues (Sims, Jacobs, \& Knill, 2011, 2012), which considers the distortion (the difference in precision between input and output signals) and the quantity of information that can be processed by a memory system to be fundamental determinants of memory performance.

\subsubsection{Memory capacity}

In line with Miller's magical number 7, Lisman and Idiart (1995) considered that human subjects can store approximately seven short-term memories, and proposed that short-term memories are stored in oscillatory patterns of neuronal activity in brain networks supporting memory. Specifically, they described how memories might be stored in different high-frequency (40-60 Hz, a.k.a. gamma) subcycles of a low-frequency oscillation ( $5-12 \mathrm{~Hz}$, a.k.a. theta). The number of cycles of high-frequency oscillations that can be nested in the low-frequency oscillation determines the number of different memories that can be maintained simultaneously in short-term memory (with a maximum at around 7). Interestingly, their model showed that pre-existing long-term memories of individual items increase our ability to accurately store such lists in short-term memory. In our paradigm, potential goal locations are always visible during encoding and recall, so that one can consider that the longterm memory representations for these locations are available to maintain the ability of the network to store the goal location(s) in short-term memory. Here, we propose that the precision of the neural coding of information in short-term memory contributes to variations in memory capacity. This view is consistent with electrophysiological recordings in monkeys showing that visual capacity limitations appear immediately upon stimulus encoding, in a bottom-up manner (Buschman, Siegel, Roy, \& Miller, 2011).

Accordingly, our behavioral data suggest that human subjects' memory for individual locations is accurate but imprecise. Consistent with our observation is the finding that the neural coding of individual locations is necessarily imprecise (Buzsaki \& Moser, 2013). Electrophysiological recordings of rodent hippocampal place cells exhibit a graded response with a maximal, peak firing rate at the center of their place field, and weaker firing rates at the periphery of their place fields. Moreover, there is some overlap between the neural representations of different locations, which are coded at the population level. The time delays between overlapping place cells (cells firing at approximately the same location) within a theta cycle are proportional to the time it takes for the animal to travel between the peaks of their place fields, and thus limit the number of cell assemblies that can be distinguished within a theta cycle. In turn, the duration of the theta cycle limits the distances that can be linearly resolved by the sequential activity of hippocampal neurons in a given environment. Accordingly, the number of cycles of high-frequency gamma oscillations that can be nested in the low-frequency theta oscillation limits the number of locations than can be stored within a single theta cycle (Buzsaki \& Moser, 2013; Lisman \& Jensen, 2013), thus, delimiting the number of different spatial locations that can be maintained simultaneously in short-term memory. Thus, converging evidence from electrophysiological, behavioral and modeling experiments indicate that, at least in the spatial domain, memory precision predicts memory capacity. However, Miller's early observations, as well as our current understanding of sensory coding, suggest that this concept is likely to generalize to other modalities and memory systems. Indeed, preliminary results from our laboratory have shown that when participants are asked to recall colors seen on trial-unique basis, when they err, they make within-shade errors (i.e., choosing a different shade of the same color) rather than across-color errors (i.e., choosing a different color altogether). This error pattern suggests imprecise coding of single 
stimuli, which, as our model suggests, can lead to limitations in memory capacity. This hypothesis will require further investigation.

\subsection{Conclusion}

We found no magical number for human short-term spatial memory capacity in a real-world setting. Instead, we found that memory performance for one location can be used to predict memory performance for multiple locations. Our findings are consistent with a theoretical model suggesting that the precision of the memory for individual locations determines the capacity of short-term memory for spatial information.

\section{Acknowledgments}

This research was supported by the Swiss National Science Foundation: grants PMPDP3-122844, PMPDP3-128996 to PBL and grants PP00A-106701, PP00P3-124536 to PL. The Swiss National Science Foundation had no involvement in the study design, nor in the collection, analysis or interpretation of the data, nor in the writing of this report or the decision to submit it for publication. The authors have not conflicts of interest to declare. The authors would like to thank the participants of this study, and Farfalla Ribordy for technical assistance.

\section{References}

Abrahams, S., Pickering, A., Polkey, C. E., \& Morris, R. G. (1997). Spatial memory deficits in patients with unilateral damage to the right hippocampal formation. Neuropsychologia, 35(1), 11-24.

Avons, S. E. (2007). Spatial span under translation: A study of reference frames. Memory \& Cognition, 35(3), 402-417.

Baddeley, A. (2003). Working memory: Looking back and looking forward. Nature Reviews Neuroscience, 4(10), 829-839. http:// dx.doi.org/10.1038/nrn1201

Baddeley, A., \& Hitch, G. J. (1974). Working memory. In G. Bower (Ed.). Recent advances in learning and motivation (Vol. 8). Academic Press.

Banta Lavenex, P., Amaral, D. G., \& Lavenex, P. (2006). Hippocampal lesion prevents spatial relational learning in adult macaque monkeys. Journal of Neuroscience, 26(17), 4546-4558.

Banta Lavenex, P., Colombo, F., Ribordy Lambert, F., \& Lavenex, P. (2014). The human hippocampus beyond the cognitive map: Evidence from a densely amnesic patient. Frontiers in Human Neuroscience, 8, 711. http://dx.doi.org/10.3389/ fnhum.2014.00711.

Banta Lavenex, P., \& Lavenex, P. (2009). Spatial memory and the monkey hippocampus: Not all space is created equal. Hippocampus, 19(1), 8-19. http://dx.doi.org/10.1002/hipo.20485.

Banta Lavenex, P., Lecci, S., Pretre, V., Brandner, C., Mazza, C., Pasquier, J., et al (2011). As the world turns: Short-term human spatial memory in egocentric and allocentric coordinates. Behavioural Brain Research, 219(1), 132-141. http://dx.doi.org 10.1016/j.bbr.2010.12.035. S0166-4328(11)00022-2 [pii].

Bays, P. M., \& Husain, M. (2008). Dynamic shifts of limited working memory resources in human vision. Science, 321(5890) 851-854. http://dx.doi.org/10.1126/science.1158023.

Becker, J. T., \& Morris, R. G. (1999). Working memory(s). Brain and Cognition, 41(1), 1-8. http://dx.doi.org/10.1006 brcg.1998.1092.

Berch, D. B., Krikorian, R., \& Huha, E. M. (1998). The Corsi block-tapping task: Methodological and theoretical considerations. Brain and Cognition, 38(3), 317-338. http://dx.doi.org/10.1006/brcg.1998.1039.

Burgess, N. (2006). Spatial memory: How egocentric and allocentric combine. Trends in Cognitive Sciences, 10(12), 551-557.

Burgess, N., Spiers, H. J., \& Paleologou, E. (2004). Orientational manoeuvres in the dark: Dissociating allocentric and egocentric influences on spatial memory. Cognition, 94(2), 149-166.

Buschman, T. J., Siegel, M., Roy, J. E. \& Miller, E. K. (2011). Neural substrates of cognitive capacity limitations. Proceedings of the National Academy of Sciences of the United States of America, 108(27), 11252-11255. http://dx.doi.org/10.1073/ pnas. 1104666108

Buzsaki, G., \& Moser, E. I. (2013). Memory, navigation and theta rhythm in the hippocampal-entorhinal system. Nature Neuroscience, 16(2), 130-138. http://dx.doi.org/10.1038/nn.3304.

Corsi, P. M. (1972). Human memory and the medial temporal lobe region of the brain. Montreal: McGill University.

Cowan, N. (2001). The magical number 4 in short-term memory: A reconsideration of mental storage capacity. Behavioral and Brain Sciences, 24(1), 87-114. discussion 114-185.

Cowan, N. (2008). What are the differences between long-term, short-term, and working memory? Progress in Brain Research, 169, 323-338. http://dx.doi.org/10.1016/S0079-6123(07)00020-9.

Donkin, C., Nosofsky, R. M., Gold, J. M., \& Shiffrin, R. M. (2013). Discrete-slots models of visual working-memory response times. Psychological Review, 120(4), 873-902. http://dx.doi.org/10.1037/a0034247.

Fagot, J., \& De Lillo, C. (2011). A comparative study of working memory: Immediate serial spatial recall in baboons (Papio papio) and humans. Neuropsychologia, 49(14), 3870-3880. http://dx.doi.org/10.1016/j.neuropsychologia.2011.10.003. 
Farrell Pagulayan, K., Busch, R. M., Medina, K. L., Bartok, J. A., \& Krikorian, R. (2006). Developmental normative data for the Corsi Block-tapping task. Journal of Clinical and Experimental Neuropsychology, 28(6), 1043-1052. http://dx.doi.org/10.1080/ 13803390500350977.

Gorgoraptis, N., Catalao, R. F., Bays, P. M., \& Husain, M. (2011). Dynamic updating of working memory resources for visual objects. The Journal of Neuroscience: The Official Journal of the Society for Neuroscience, 31(23), 8502-8511. http://dx.doi.org/ 10.1523/JNEUROSCI.0208-11.2011.

Holdstock, J. S., Mayes, A. R., Cezayirli, E., Aggleton, J. P., \& Roberts, N. (1999). A comparison of egocentric and allocentric spatial memory in medial temporal lobe and korsakoff amnesics. Cortex, 35, 479-501.

Holdstock, J. S., Mayes, A. R., Cezayirli, E., Isaac, C. L., Aggleton, J. P., \& Roberts, N. (2000). A comparison of egocentric and allocentric spatial memory in a patient with selective hippocampal damage. Neuropsychologia, 38(4), 410-425.

Lisman, J. E., \& Idiart, M. A. (1995). Storage of $7 \pm 2$ short-term memories in oscillatory subcycles. Science, 267(5203), $1512-1515$.

Lisman, J. E., \& Jensen, O. (2013). The theta-gamma neural code. Neuron, 77(6), 1002-1016. http://dx.doi.org/10.1016 j.neuron.2013.03.007.

Luck, S. J., \& Vogel, E. K. (1997a). The capacity of visual working memory for features and conjunctions. Nature, 390(6657), 279-281. http://dx.doi.org/10.1038/36846.

Luck, S. J., \& Vogel, E. K. (1997b). The capacity of visual working memory for features and conjunctions. Nature, 390(6657), 279-281. http://dx.doi.org/10.1038/36846.

Ma, W. J., Husain, M., \& Bays, P. M. (2014). Changing concepts of working memory. Nature Neuroscience, 17(3), 347-356. http:/ dx.doi.org/10.1038/nn.3655.

Miller, G. A. (1956). The magical number seven plus or minus two: Some limits on our capacity for processing information. Psychological Review, 63(2), 81-97.

Morris, R. G., Pickering, A., Abrahams, S., \& Feigenbaum, J. D. (1996). Space and the hippocampal formation in humans. Brain Research Bulletin, 40(5-6), 487-490.

Mou, W., McNamara, T. P., Valiquette, C. M., \& Rump, B. (2004). Allocentric and egocentric updating of spatial memories. Journal of Experimental Psychology. Learning, Memory, and Cognition, 30(1), 142-157. http://dx.doi.org/10.1037/0278-7393.30.1.142.

O’Keefe, J., \& Nadel, L. (1978). The hippocampus as a cognitive map. Oxford: Clarendon Press.

Parmentier, F. B., Elford, G., \& Mayberry, M. (2005). Transitional information in spatial serial memory: Path characteristics affect recall performance. Journal of Experimental Psychology. Learning, Memory, and Cognition, 31(3), 412-427. http://dx.doi.org/ 10.1037/0278-7393.31.3.412.

Piccardi, L., Bianchini, F., Argento, O., De Nigris, A., Maialetti, A., Palermo, L., et al (2013). The Walking Corsi Test (WalCT): Standardization of the topographical memory test in an Italian population. Neurological Sciences, 34(6), 971-978. http:// dx.doi.org/10.1007/s10072-012-1175-x.

Piccardi, L., Iaria, G., Ricci, M., Bianchini, F., Zompanti, L., \& Guariglia, C. (2008). Walking in the Corsi test: Which type of memory do you need? Neuroscience Letters, 432(2), 127-131. http://dx.doi.org/10.1016/j.neulet.2007.12.044.

Rouder, J. N., Morey, R. D., Cowan, N., Zwilling, C. E., Morey, C. C., \& Pratte, M. S. (2008). An assessment of fixed-capacity models of visual working memory. Proceedings of the National Academy of Sciences of the United States of America, 105(16), 5975-5979. http://dx.doi.org/10.1073/pnas.0711295105.

Simons, D. J., \& Wang, R. F. (1998). Perceiving real-world viewpoint changes. Psychological Science, 9(4), 315-320.

Sims, C. R., Jacobs, R. A., \& Knill, D. C. (2011). Adaptive allocation of vision under competing task demands. The Journal of Neuroscience: The Official Journal of the Society for Neuroscience, 31(3), 928-943. http://dx.doi.org/10.1523/JNEUROSCI.424010.2011 .

Sims, C. R., Jacobs, R. A., \& Knill, D. C. (2012). An ideal observer analysis of visual working memory. Psychological Review, 119(4), 807-830. http://dx.doi.org/10.1037/a0029856.

Unsworth, N., \& Engle, R.W. (2007). On the division of short-term and working memory: An examination of simple and complex span and their relation to higher order abilities. Psychological Bulletin, 133(6), 1038-1066. http://dx.doi.org/10.1037/00332909.133.6.1038.

van den Berg, R., Shin, H., Chou, W. C., George, R., \& Ma, W. J. (2012). Variability in encoding precision accounts for visual shortterm memory limitations. Proceedings of the National Academy of Sciences of the United States of America, 109(22), 8780-8785. http://dx.doi.org/10.1073/pnas.1117465109.

Wang, R. F., \& Simons, D. J. (1999). Active and passive scene recognition across views. Cognition, 70(2), 191-210.

Zhang, W., \& Luck, S. J. (2008). Discrete fixed-resolution representations in visual working memory. Nature, 453(7192), $233-235$. http://dx.doi.org/10.1038/nature06860. 